\title{
Protocol Deviations Domain
}

National Cancer Institute

\section{Source}

National Cancer Institute. Protocol Deviations Domain. NCI Thesaurus. Code C49585.

A subject domain utilized for the submission of information encompassing and representing data, vocabulary or records related to protocol deviations. 